\title{
Erratum: Directly Detecting MeV-Scale Dark Matter via Solar Reflection [Phys. Rev. Lett. 120, 141801 (2018)]
}

\author{
Haipeng An, Maxim Pospelov, Josef Pradler, and Adam Ritz \\ (Received 16 November 2018; published 18 December 2018)
}

DOI: 10.1103/PhysRevLett.121.259903

In this Letter, we pointed out that additional direct detection sensitivity to dark matter (DM) in the $10 \mathrm{keV}-10 \mathrm{MeV}$ mass range is possible through consideration of "reflected DM" initially scattered by more energetic electrons in the Sun (or Earth) prior to scattering in the terrestrial underground detector.

As part of the calculation required to determine the reflected flux, the probability for a DM particle to scatter with a solar electron is determined according to its mean free path $l_{\mathrm{fp}}(r)$ at each radial location $r$ within the Sun. In this Erratum, we correct the calculation to fully account for the dependence of $l_{\mathrm{fp}}(r)$ on the velocity $v_{r}$, the DM particle relative to the electron in each scattering event, i.e., $l_{\mathrm{fp}}(r)=\left[n_{e}(r)\left\langle\sigma_{e} v_{r}\right\rangle\right]^{-1} \times v_{\mathrm{DM}}$. This leads to a revised reflected DM energy spectrum in Fig. 3, and associated revisions to the constraint contours in Fig. 2. Updated results are presented in Fig. 1, and further details are contained in the Supplemental Material of the Letter. The conclusions of the Letter are otherwise unchanged.
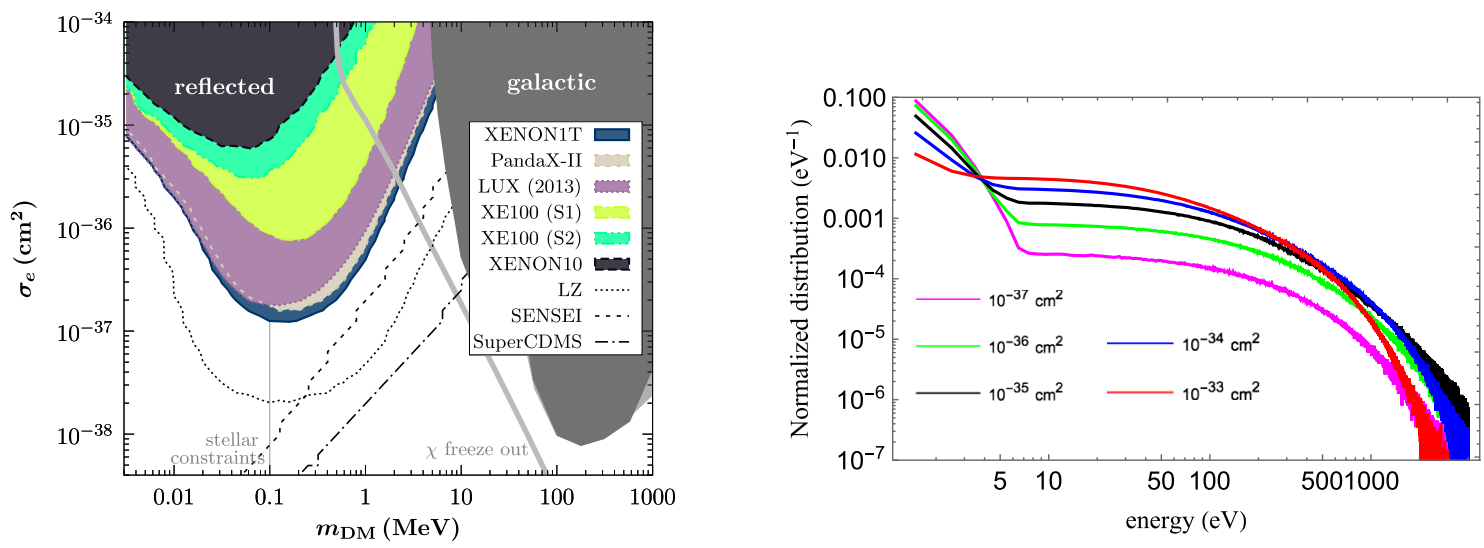

FIG. 1. (Left) Corrected version of Fig. 2 showing exclusion contours for reflected DM from a range of experiments, in comparison to previous limits from XENON10 and XENON100 on scattering from the "galactic DM" halo population. (Right) Corrected version of Fig. 3 showing the normalized energy distributions for reflected DM with a mass of $3 \mathrm{MeV}$ and the range of scattering cross sections indicated. (See the captions of Figs. 2 and 3 in the Letter for further details).

We thank Niklas Nielsen and Timon Emken for pointing out the need for this more refined treatment of the DM mean free path in the Sun.

Published by the American Physical Society under the terms of the Creative Commons Attribution 4.0 International license. Further distribution of this work must maintain attribution to the author(s) and the published articles title, journal citation, and DOI. 